\title{
Picosecond Flash Photolysis of Photochromic Spiropyrans
}

\author{
S. A. KRYSANOV and M. V. ALFIMOV \\ Institute of Chemical Physics, USSR Academy of Sciences, 142432 Cher- \\ nogolovka, Moscow Region, USSR
}

(Received February 16, 1983)

\begin{abstract}
The transient species involved in photocoloration and photobleaching of indoline 6-nitrosubstituted spiropyran solutions at room temperature were studied by picosecond flash photolysis. Transient absorption spectrum of spiropyran colorless form consists of two bands peaked at 440 and $585 \mathrm{~nm}$ and is assigned to the photoinduced intermediate non-planar cis configuration with the breaked $\mathrm{C}-\mathrm{O}$ bond of the pyran ring. This transient is found to have the buildup time shorter than $10 \mathrm{ps}$ and initial decay times of about $10 \mathrm{~ns}$ in benzene and $5 \mathrm{~ns}$ in ethanol. The colored form exhibits the transient absorption peaked at $460 \mathrm{~nm}$ with the lifetime of about $200 \mathrm{ps}$ in ethanol which is assigned to $S_{n} \leftarrow S_{1}$ absorption of colored form. The predominant decay channel of the colored form $S_{1}$ state is shown to be internal conversion $S_{1} \leadsto S_{0}$ with the quantum yield of about $0.70 \pm 0.15$.
\end{abstract}

\section{INTRODUCTION}

Spiropyrans belong to the most important class of the organic photochromic compounds which are used in the light sensitive materials. ${ }^{1}$ Intensive study of the spiropyran photochromism during three decades $^{2}$ has allowed us to appreciate the general photochemical reaction (for reviews see Refs. 3-5). The initial colorless form A with the orthogonal planes of two molecular halves suffer $\mathrm{C}-\mathrm{O}$ bond breaking of the pyran ring under ultraviolet light. The final planar colored form B absorbing at about $600 \mathrm{~nm}$ is the mixture of four conformers of the trans isomer differing by the rotation angle about single bonds of the polymethene chain.

Though the structure of $\mathrm{A}$ and $\mathrm{B}$ forms is well established, there is no common point of view on the detailed mechanism of the photochromic reaction. The detailed mechanism is discussed usually ${ }^{4}$ 


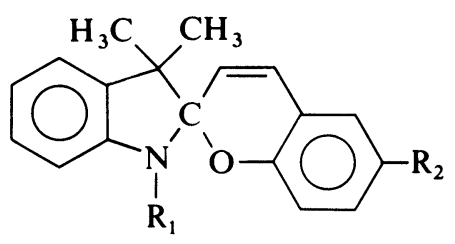

A

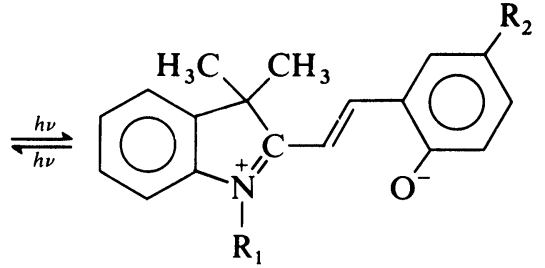

B

in terms of the intermediate $c i$ is isomer $\mathrm{X}^{2}$ The $\mathrm{X}$ isomer represents the photoinduced non-planar configuration with breaked $\mathrm{C}-\mathrm{O}$ bond. Such intermediate must exist in both photochromic processes: in photocoloration, $A \rightarrow B$, after $\mathrm{C}-\mathrm{O}$ bond breaking, and in the photobleaching, $B \rightarrow A$, before $\mathrm{C}-\mathrm{O}$ bond restoration.

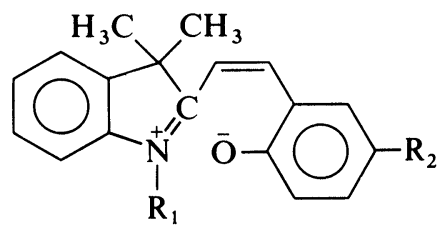

$\mathrm{X}$

Much attention was given to the indoline nitrosubstituted $\left(\mathbf{R}_{\mathbf{2}}=\right.$ $\mathrm{NO}_{2}$ ) spiropyrans having a largest photocoloration efficiency. ${ }^{4}$ The transient absorption band with a peak at $440 \mathrm{~nm}$ with a lifetime of about $10^{-7} \mathrm{~s}$ was observed ${ }^{6}$ in the nanosecond studies of these compounds. This transient was founded to be formed both in photocoloration and photobleaching and was ascribed ${ }^{6}$ to the $\mathrm{X}$ isomer. The $440 \mathrm{~nm}$ band was ascribed to the triplet state of form $A$ in other works. ${ }^{7}$ The transient species buildup was studied in Ref. 8 by means of the streak camera. The buildup times of the photoinduced final B $(590 \mathrm{~nm})$ and intermediate $(440 \mathrm{~nm})$ absorption bands were shown ${ }^{8}$ to be synchronous within several nanosecond and to coincide with the time resolution of the streak camera used. These results show that methods with the time resolution less than $10^{-9} \mathrm{~s}$ are needed for the primary processes study in the spiropyrans photochromic transformations. 
In this paper we present experimental results on picosecond flash photolysis of the indoline nitrosubstituted spiropyran $S P_{1}\left(\mathrm{R}_{1}=\mathrm{CH}_{3}\right.$; $\mathrm{R}_{2}=\mathrm{NO}_{2}$ ).

\section{EXPERIMENTAL}

An undegassed solution of $S P_{1}$ in benzene and ethanol $\left(10^{-3} \mathrm{M}\right)$ in a $1 \mathrm{~mm}$ quartz cell at $295 \mathrm{~K}$ were used in the photo-coloration study. The ethanol solution of $S P_{1}$ was used in the photobleaching study since the thermostability of form B is increased considerably in polar solvents. The colored form was prepared by illumination of the solution of the initial form with the $265-313 \mathrm{~nm}$ lines of a $1000 \mathrm{~W}$ mercury lamp. The solution was illuminated before every laser shot to restore the form $B$ concentration.

The apparatus used to measure picosecond transient absorbance spectra was the same as that described in detail elsewhere. ${ }^{9}$ Briefly, it includes a mode-locked Nd:phosphate glass laser on the basis of an unstable resonator configuration and amplifier system to produce single $6 \mathrm{ps}, \mathrm{TEM}_{00}$ pulses at $1055 \mathrm{~nm}$. The second $528 \mathrm{~nm}$ (an energy $1 \mathrm{~mJ}$ ) and third $352 \mathrm{~nm}$ (an energy $0.5 \mathrm{~mJ}$ ) harmonics were used as excitation pulses. For probing, the $1055 \mathrm{~nm}$ pulse generated in $\mathrm{D}_{2} \mathrm{O}$ a broad-band continuum. The double-beam optical system consisting of the semi-transparent $\mathrm{Al}$ mirror and five miniature mirrors forms interrogating continuum pulses delayed by $10 \mathrm{ps}, 400 \mathrm{ps}, 1 \mathrm{~ns}, 2.5 \mathrm{~ns}$, $5 \mathrm{~ns}$ with respect to the excitation pulse. The absorbance changes $\Delta A$ in a sample were detected photographically and calculated for each time using two alternate laser shots in presence $(p)$ and absence $(a)$ of the excitation pulse:

$$
\Delta A=\gamma^{-1}\left[\left(D_{1}^{p}-D_{2}^{p}\right)-\left(D_{1}^{a}-D_{2}^{a}\right)\right]
$$

where $\gamma$ and $D$ are the contrast and blackening of the film.

\section{RESULTS AND DISCUSSION}

The transient absorption with two peaks at 440 and $585 \mathrm{~nm}$ was detected on flashing the benzene solution of colorless form with a $352 \mathrm{~nm}$ picosecond pulse, as shown in Figure 1. The spectra measured 


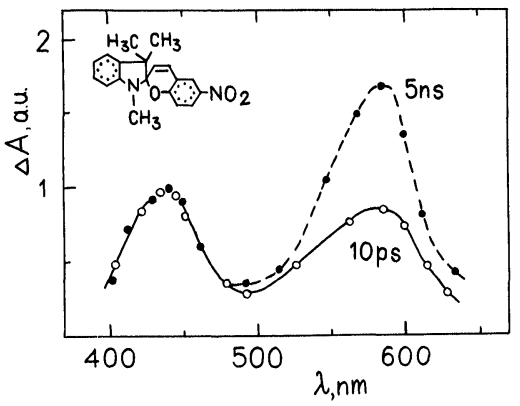

FIGURE 1 Transient absorbance spectra of the colorless form $S P_{1}$ in benzene at $295 \mathrm{~K}$ measured at $10 \mathrm{ps}$ and $5 \mathrm{~ns}$ delays after excitation with a $6 \mathrm{ps}, 352 \mathrm{~nm}$ pulse.

at 10 ps and $5 \mathrm{~ns}$ delays were noramlized with respect to the shortwavelength band in this Figure. The shape and maximum position of these bands as seen do not depend on time. An absorbance increase of the long-wavelength band was only observed in the time region studied. The kinetic behavior of 440 and $585 \mathrm{~nm}$ bands in the benzene solution is shown in Figure 2. The angle between the polarization planes of the excitation and probe beams was arbitrary in this case, and the slow buildup during $400 \mathrm{ps}$ is due to rotation relaxation. The transient absorption band $440 \mathrm{~nm}$ is seen to decay with the characteristic time of about $10 \mathrm{~ns}$. The initial parts of the kinetic curves for the 440 and $585 \mathrm{~nm}$ bands coincide in the $10 \mathrm{ps}-2 \mathrm{~ns}$ region and the increase of the $585 \mathrm{~nm}$ band absorbance is observed for the later delay times.

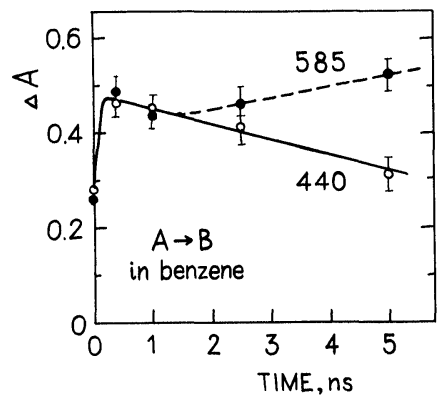

FIGURE 2 Absorbance kinetics at 440 and $585 \mathrm{~nm}$ of the $S P_{1}$ colorless form in benzene. The point with the zero absciss refers to the $10 \mathrm{ps}$ delay time. Excitation with a $352 \mathrm{~nm}$ picosecond pulse when rotational relaxation is not eliminated. 
An analogous transient absorption bands with the same maxima at 440 and $585 \mathrm{~nm}$ were observed for the ethanolic solution of the $S P_{1}$ colorless form. The only difference was that the spectra width for the ethanolic solution was larger than for the benzene one. The kinetic behavior of these bands in the ethanolic solution is shown in Figure 3. The rotational relaxation effects were eliminated here due to a proper adjustment of the angle between polarization planes $\left(54.7^{\circ}\right) .{ }^{10}$ Both transient bands are formed within the duration of the 6 ps excitation pulse and its optical density diminishes in the beginning with the same characteristic time of about $5 \mathrm{~ns}$. The kinetic curves change later, namely, the long-wavelength band absorbance tends to increase and the short-wavelength band decay becomes slower.

The results of the $S P_{1}$ colored form study are represented in Figures 4 and 5 . Excitation of a form $B$ solution with a $528 \mathrm{~nm}$ pulse resulted in the immediate bleaching (negative $\Delta A$, Figure 4) of the groundstate absorption band at $550 \mathrm{~nm}$. The transient absorption band with the peak at $460 \mathrm{~nm}$ was formed simultaneously with the diminution of the $550 \mathrm{~nm}$ band. The $460 \mathrm{~nm}$ band decays completely within $1 \mathrm{~ns}$ (Figure 5). The recovery of the bleached $550 \mathrm{~nm}$ band occurs in the same time region. It is worthwhile mentioning that the $550 \mathrm{~nm}$ recovery is not complete and the stationary value $\Delta A_{550}$ forms $25 \%$ of the initial value measured at $10 \mathrm{ps}$.

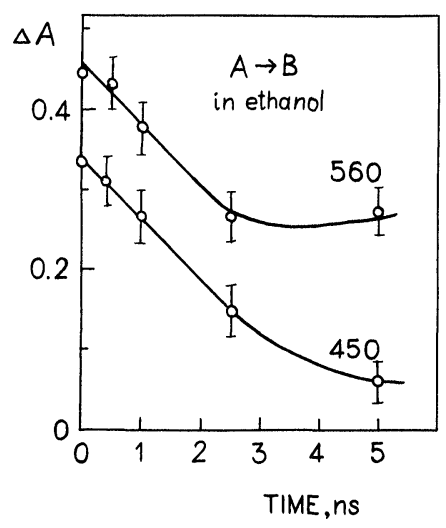

FIGURE 3 Absorbance kinetics at 450 and $560 \mathrm{~nm}$ of the $S P_{1}$ colorless form in ethanol. Excitation with a $352 \mathrm{~nm}$ picosecond pulse under conditions of the rotational relaxation elimination. 


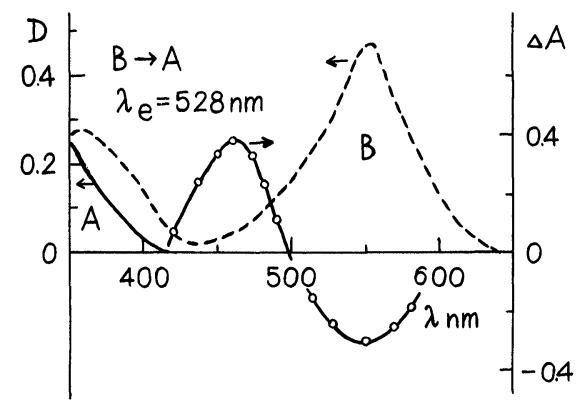

FIGURE 4 Spiropyran $S P_{1}$ in ethanol at $295 \mathrm{~K}$. Right-hand scale: ground-state absorption spectra of colorless A and colored B forms. Left-hand scale: transient absorbance spectrum of colored form B (open circles) measured at $10 \mathrm{ps}$ delay after excitation with a $528 \mathrm{~nm}$ picosecond pulse.

Let us consider the assignment of the states which exhibit the transient absorption. The short-wavelength band peak $440 \mathrm{~nm}$ coincides exactly with that in the nanosecond experiments. ${ }^{6-8}$ This coincidence proves the identity of the transient species observed in nano- and pico-second flash photolysis experiments. As pointed out in the Introduction, the $440 \mathrm{~nm}$ is ascribed at present to two species: to the triplet state of the colorless form $\mathrm{A}^{7}$ and to the cis isomer $\mathrm{X}^{6}$ The data presented in Figures 2 and 3 entitle us to assign the transient absorption band $440 \mathrm{~nm}$ to the $\mathrm{X}$ isomer. Indeed, the lifetime of the

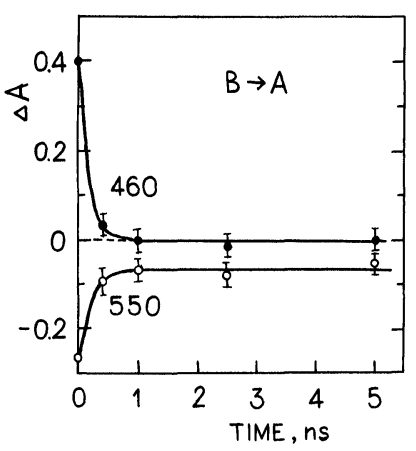

FIGURE 5 Absorbance kinetics at 460 and $550 \mathrm{~nm}$ of the $S P_{1}$ colored form in ethanol. Excitation with a $528 \mathrm{~nm}$ picosecond pulse under conditions of the rotational relaxation elimination. 
form A triplet state in solid solutions at $7 \mathrm{~K}$ is of the order $0.1 \mathrm{~s} .{ }^{11}$ This lifetime in the room temperature solution can be diminished due to the quenching by oxygen to $\approx 2 \cdot 10^{-7} \mathrm{~s}$ at the usual oxygen concentration $10^{-3} \mathrm{M}$ and diffusion-controlled quenching rate in ethanol $5 \cdot 10^{9} \mathrm{M}^{-1} \mathrm{~s}^{-1}$. The measured times of the $440 \mathrm{~nm}$ band decay of about $10^{-8} \mathrm{~s}$ are much less than the triplet state lifetimes. Combining this result with that in nanosecond experiments ${ }^{6}$ we thus conclude that the $\mathrm{X}$ isomer decay is characterized by two decay times of about $10^{-8}$ and $10^{-7} \mathrm{~s}$.

The fast decay was observed not only for the $440 \mathrm{~nm}$ band but also for the $585 \mathrm{~nm}$ band. The coincidence of the initial parts of the kinetic curves for these bands indicate that both bands belong to the same intermediate isomer $\mathrm{X}$. The fast formation of the long-wavelength band within the $20 \mathrm{~ns}$ pulse duration was interpreted in the nanosecond experiments ${ }^{6}$ as the photocoloration occuring directly via the singlet state $S_{1}$ of form A without the intermediate form X involvement. The above assignment of the $585 \mathrm{~nm}$ band allows us to explain the photocoloration process in terms of only one primary transient, namely the $\mathrm{X}$ form. This assignment is supported further by the fact that the transient $585 \mathrm{~nm}$ band maximum differs from that of the ground-state absorption spectrum of form B at $550 \mathrm{~nm}$ in ethanol (Figure 4). The contribution of the final form $B$ begins to manifest for delays more than 3-5 ns (Figures 2 and 3).

The present results reveal two properties of the transient $\mathrm{X}$ which have to be elucidated, namely the ultrafast formation of the $\mathrm{X}$ isomer and its two-component decay. Let us consider the known from the literature photoprocesses in spiropyrans to interpret the ultrafast $\mathrm{X}$ formation. The indoline and pyrans parts absorb approximately independently and the transfer energy between these parts can occur. ${ }^{12,13}$ The mechanism of the spiropyrans photochromism is determined first of all by the relative position of the energy levels. The indoline nitrosubstituted spiropyran $S P_{1}$ has the following state ordering: $S_{0}, T_{\pi \pi^{*}}, T_{n \pi^{*}}, S_{\pi \pi^{*}}, S_{n \pi^{*}}{ }^{13}$ The long-wavelength absorption and phosphorescence are due to $\pi \pi^{*}$-transitions ${ }^{13}$ in pyran part of form A. Since the energy level of the $T_{n \pi^{*}}$ state is lower than the $S_{\pi \pi^{*}}$ state, the intersystem crossing probability is high $\left(10^{11} \mathrm{~s}^{-1}\right)$ according to the systematization ${ }^{14}$ that results in both fluorescence quenching and effective population of the triplet state. An ultrafast formation of the transient $\mathrm{X}$ can thus be explained by the photodissociation 
from the lowest triplet state of form $A$. This process would imply an absence of the form A phosphorescence. But the fact is form A possesses a phosphorescence $e^{3,12}$ and we have to conlude that the transient $\mathrm{X}$ formation must occur via an upper triplet state of form A. An ultrafast internal conversion between an upper triplet level can compete in this case with an ultrafast dissociation that results in the population of the phosphorscent level. This conclusion is in agreement with the results of the sensitized photocoloration experiments. ${ }^{3,15-17}$ The presence of two kinds of triplet levels, phosphorescent and photochemically active, with the energy difference of about $1500 \mathrm{~cm}^{-1}$ was shown in these works. In our opinion, a some upper vibrational level of the lowest $T_{\pi \pi^{*}}$ state can be responsible for the photochemical active level since the energy difference between the triplet $\pi \pi^{*}$ and $n \pi^{*}$ states of the pyran ring is of about $5000 \mathrm{~cm}^{-1} .^{13}$

The existence of two characteristic times of the isomer $\mathrm{X}$ decay allows us to choose the following kinetic scheme from the many one proposed for the photochromic processes analysis ${ }^{9} \mathrm{XXX}$ :

$$
\mathrm{A} \underset{k_{1}}{\stackrel{k_{0}}{\rightleftharpoons}} \mathrm{X} \underset{k_{3}}{\stackrel{k_{2}}{\rightleftharpoons}} \mathrm{B}_{1} \stackrel{k_{4}}{\longrightarrow} \mathrm{B}_{2,3,4}
$$

where $\mathrm{B}_{1}$ is the least stable trans isomer of the colored form, $\mathrm{B}_{2,3,4}$ are the rest of the trans iomers with the increasing stability. We have for the times longer than $k_{0}^{-1}=10 \mathrm{ps:}$

$$
\begin{gathered}
X=\alpha \exp \left(-\lambda_{1} t\right)+\beta \exp \left(-\lambda_{2} t\right) \\
\lambda_{1,2}=\frac{1}{2}\left(k_{1}+k_{2}+k_{3}+k_{4}\right) \mp\left[\left(k_{1}+k_{2}-k_{3}-k_{4}\right)^{2}+4 k_{2} k_{3}\right]^{1 / 2}
\end{gathered}
$$

For the ethanolic solution $\lambda_{1}=3 \cdot 10^{6} \mathrm{~s}^{-1}[6], \lambda_{2}=2 \cdot 10^{8} \mathrm{~s}^{-1}$. From the conditions $\lambda_{1} \ll \lambda_{2}$ and $k_{1}+k_{2}+k_{3}+k_{4}=\lambda_{1}+\lambda_{2}$ we obtained

$$
\left(1+\frac{k_{1}}{k_{2}}\right)\left(1+\frac{k_{4}}{k_{3}}\right) \approx 1
$$

that is satisfied at $k_{2} \gg k_{1}$ and $k_{3} \gg k_{4}$. We suppose further $k_{3}<k_{2}$ since the process $B_{1} \rightarrow X$ is connected with the potential barrier overcoming. Indeed, the $X$ form has an energy of about $10^{4} \mathrm{~cm}^{-14}$ higher than the $\mathrm{B}$ forms.

We conclude from these estimates that (1) the transformation of the cis isomer $\mathrm{X}$ into the least stable trans isomer of form $\mathrm{B}$ is responsible for the fast $\mathrm{X}$ decay with $k_{2} \simeq 10^{8} \mathrm{~s}^{-1}$; (2) large efficiency ${ }^{12}$ 
If the photocoloration in the indoline nitrosubstituted spiropyrans ${ }^{4}$ is due to the fast transition of the $\mathrm{X}$ isomer into the colored form $\left(\mathrm{X} \rightarrow \mathrm{B}_{1}\right)$ in comparison with the back process $(\mathrm{X} \rightarrow \mathrm{A})$.

As for the transients in the photobleaching $\mathrm{B} \rightarrow \mathrm{A}$ process, we assign the transient absorption band $460 \mathrm{~nm}$ (Figure 4) to the $S_{n} \leftarrow S_{1}$ transition from the lowest excited singlet state of the colored form. Indeed, the $460 \mathrm{~nm}$ band is formed for the time shorter than $10 \mathrm{ps}$ and its decay time $200(100 \mathrm{ps})$ is close to the flurorescence lifetime of form B ( $\left.400 \mathrm{ps}^{\text {in ethanol }}{ }^{3}\right)$. Moreover, the $460 \mathrm{~nm}$ band decay is accompanied by recovery of the ground-state absorption band $550 \mathrm{~nm}$ in the same time region. An incomplete recovery of the $550 \mathrm{~nm}$ band is ascribed naturally to an intersystem crossing in form $B$ and the above given value $25 \%$ for the stationary rest $\Delta A_{550}$ determines just the quantum yield of intersystem crossing. In view of the small fluorescence quantum yield $\left(0.02^{3}\right)$ we conclude that internal conversion with the quantum yield of $0.70 \pm 0.15$ is the predominant decay channel of the lowest excited singlet state $S_{1}$ of form B. An absence of the $\mathrm{X}$ form absorption in the time region up to $5 \mathrm{~ns}$ is due to its formation probably via the long-lived triplet state of form B.

\section{References}

1. V. A. Barachevsky, G. I. Lashkov and V. A. Tzekhomsky, Photochromism i ego primenenie (Khimiya, Moscow, 1977).

2. E. Fischer and Y. Hirschberg, J. Chem. Soc. 4522, (1952).

3. G. I. Lashkov, M. V. Savostjanova, A. V. Shablya and T. A. Shakhverdov, in: Molekulyarnaya Photonika (Nauka, Leningrad, 1970) p. 299.

4. R. C. Bertelson, in: Techniques of Chemistry: Photochromism, Vol. 3, ed. G. M. Brown (Wiley-Interscience, New York, 1971) p. 45.

5. A. S. Kholmansky, A. V. Zubkov and K. M. Dyumaev, Uspekhi Khim. 50, 569, (1981).

6. V. A. Murin, V. F. Mandgikov and V. A. Barachevsky, Opt. i Spektrosk. 37, 1174, (1974).

7. Y. Kalisky and D. J. Williams, Chem. Phys. Lett. 86, 100, (1982).

8. S. G. Kuzmin, V. F. Mandgikov, V. A. Barachevsky, E. P. Pozhidaev and E. A. Kuzmina, Abstracts of the 14th Congress of High-Speed Photography and Photonics, Moscow (1980) p. 353.

9. S. A. Krysanov and M. V. Alfimov, Chem. Phys Lett. 76, 221, (1980); Chem. Phys. Lett. in press.

10. H. E. Lessing and A. von Jena, Chem. Phys. Lett. 42, 213, (1976).

11. T. A. Shakhverdov and A. V. Shablya, Opt. i Spektrosk. 24, 619, (1968).

12. N. W. Tyer and R. S. Becker, JACS 92, 1295, (1970).

13. I. L. Belaiz, T. D. Platonova and V. A. Barachevsky, Opt. i Spektrosk. 49, 1125, (1980). 
14. R. N. Nurmukhametov, V. G. Plotnikov and D. N. Shigorin, Z. Fiz. Khim. 40, 1154, (1966).

15. T. Bercovici and E. Fischer, JACS 86, 5687, (1964).

16. R. S. Becker and J. K. Roy, J. Phys. Chem. 69, 1435, (1965).

17. D. A. Reeves and F. Wilkinson, J. Chem. Sod. Faraday Trans. II 69, 1381, (1973). 